\title{
Finite-momentum exciton landscape in mono- and bilayer transition metal
} dichalcogenides

\author{
Deilmann, Thorsten; Thygesen, Kristian Sommer
}

Published in:

2D materials

Link to article, DOI:

10.1088/2053-1583/ab0e1d

Publication date:

2019

Document Version

Peer reviewed version

Link back to DTU Orbit

Citation (APA):

Deilmann, T., \& Thygesen, K. S. (2019). Finite-momentum exciton landscape in mono- and bilayer transition metal dichalcogenides. 2D materials, 6(3), [035003]. https://doi.org/10.1088/2053-1583/ab0e1d

\section{General rights}

Copyright and moral rights for the publications made accessible in the public portal are retained by the authors and/or other copyright owners and it is a condition of accessing publications that users recognise and abide by the legal requirements associated with these rights.

- Users may download and print one copy of any publication from the public portal for the purpose of private study or research.

- You may not further distribute the material or use it for any profit-making activity or commercial gain

- You may freely distribute the URL identifying the publication in the public portal

If you believe that this document breaches copyright please contact us providing details, and we will remove access to the work immediately and investigate your claim 


\title{
Finite-momentum exciton landscape in mono- and bilayer transition metal dichalcogenides
}

\author{
Thorsten Deilmann ${ }^{1, \text { * }}$ and Kristian Sommer Thygesen ${ }^{1,2}$ \\ ${ }^{1}$ CAMD, Department of Physics, Technical University of Denmark, DK-2800 Kongens Lyngby, Denmark \\ ${ }^{2}$ Center for Nanostructured Graphene $(C N G)$, Technical University of Denmark, DK-2800 Kongens Lyngby, Denmark
}

(Dated: April, 5, 2019)

\begin{abstract}
Monolayers and bilayers of transition metal dichalcogenides (TMDCs) are currently being intensively scrutinized not least due to their rich opto-electronic properties which are governed by strongly bound excitons. Until now the main focus has been on excitons with zero momentum. In this study we employ $a b$ initio many-body perturbation theory within the $G W / \mathrm{BSE}$ approximation to describe the entire $Q$-resolved exciton band structure for mono- and bilayers of the $\mathrm{MX}_{2}(\mathrm{M}=\mathrm{Mo}, \mathrm{W}$ and $\mathrm{X}=\mathrm{Se}, \mathrm{S})$ TMDCs. We find that the strong excitonic effects, i.e. strong electron-hole interactions, are present throughout the entire $Q$-space. While the exciton binding energies of the lowest excitons do not vary significantly with $Q$, we find a strong variation in their coupling strength. In particular, the latter are strongly peaked for excitons at $Q=0$ and $Q=\Lambda$. For $\operatorname{MoX}_{2}$ monolayers the $\mathrm{K} \rightarrow \mathrm{K}$ ' excitons constitutes the exciton ground state, while in $\mathrm{WX}_{2}$ monolayers direct transitions at $\mathrm{K}$ are lowest in energy. Our calculations further show that the exciton landscape is highly sensitive to strain and interlayer hybridization. For all four bilayers the exciton ground state is shifted to $\Gamma \rightarrow \Lambda$ or $\mathrm{K} \rightarrow \Lambda$ transitions closely following the trends of the single-particle band structures.
\end{abstract}

\section{INTRODUCTION}

Over the past few years atomically thin twodimensional (2D) materials, in particular transition metal dichalcogenides (TMDCs) have attracted tremendous interest within several research areas including batteries, (electro-)catalysis, electronics, and photonics [1. 2]. Special attention has been given to the optoelectronic properties which can change drastically from the monolayer to the multilayer/bulk material (e.g. the transition from a direct to indirect gap semiconductor in TMDCs) 3, 4. However, until now the main focus has been on the selective valley- and spin-excitations [5 9 close to the direct gap at $K$, i.e. on excitons coupling to photons with vanishingly small momentum $Q$.

While already this small subspace of possible excitations around $Q=0$ has led to many interesting phenomena, a complete picture including excitations with nonzero momenta is only beginning to emerge. Especially for time-dependent phenomena (e.g. photoluminescence) the relative energetic alignment of zero and nonzero momentum excitons is important. Moreover, the exciton energy and coupling strength to electric field at finite $Q$ is important for modeling and understanding how excitons in TMDCs couple to localized electric fields, which is relevant for e.g. electron energy loss spectroscopy and localized plasmon-exciton coupling phenomena [10]. Only recently first principles studies on excitons with nonzero momenta have been presented [11, 12] 13. A key finding of these studies was the non-analytic dispersions of the excitons in $\mathrm{MoS}_{2}$ and $\mathrm{hBN}$ close to the $\mathrm{K}$ point, i.e. for small momentum transfer $Q$.

Furthermore, based on model calculations Feierabend

\footnotetext{
* thorsten.deilmann@wwu.de
}

et al. have proposed the usage of excitons with a non vanishing momentum for sensing and have underlined the role of such excitons for the line width and shape of the bright excitons [14 16]. However, the employed models rely on many parameters. In the present study we use $a b$ initio methods to describe the momentum resolved optical properties of the four most popular TMDCs, namely $\mathrm{MoS}_{2}, \mathrm{WS}_{2}, \mathrm{MoSe}_{2}$, and $\mathrm{WSe}_{2}$. After a short introduction to the methodology in Sec. [II we focus on the investigation of monolayer TMDCs in Sec. III as well as its bilayer form in Sec. IV.

\section{METHODS}

Our first principles investigations are carried out within the framework of density-functional theory (DFT) and many-body perturbation theory. In the DFT we employ norm-conserving pseudopotentials in the KleinmanBylander form [17, 18] and a basis of localized atomcentered Gaussian orbitals of $s, p, d$ and $s^{*}$ symmetry 19. For a detailed discussion of the many-body approach we refer to Ref. [20] and restrict ourselves here to the less conventional description of excitons with nonzero momenta. Thus we assume that we have already solved the quasi-particle Hamiltonian

$$
\hat{H}^{\mathrm{QP}} \psi_{n \mathbf{k}}=\epsilon_{n \mathbf{k}} \psi_{n \mathbf{k}}
$$

in the $G W$ or $G d W$ approximation [21, 22] and obtained the quasi-particle energies $\epsilon_{n \mathbf{k}}$ and the wave functions $\psi_{n \mathbf{k}}$ for all bands $n$ and points $\mathbf{k}$ of interest. In the next step we set up the Bethe-Salpeter equation [23 25] in the 
(a)

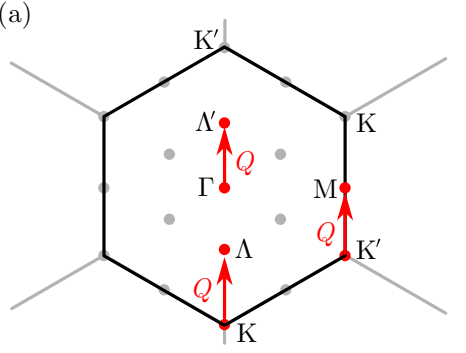

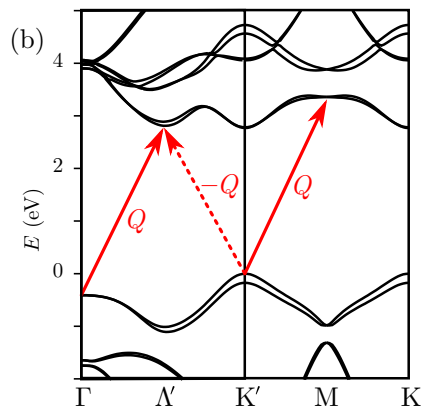

FIG. 1. (a) 2D hexagonal Brillouin zone including special points. The red arrows denote the momentum transfer $Q$. Three different transitions $\left(\Gamma \rightarrow \Lambda^{\prime}, \mathrm{K}^{\prime} \rightarrow \mathrm{M}\right.$, and $\left.\mathrm{K}^{\prime} \rightarrow \Lambda^{\prime}\right)$ are shown. We note that $Q$ and $-Q$ transitions are equivalent due to time inversion symmetry. (b) Representation of the momentum transfer $Q$ in the band structure (here: $\mathrm{MoS}_{2}$ in the $G d W$ approximation).

Tamm-Dancoff approximation

$$
\begin{aligned}
& \left(\epsilon_{c \mathbf{k}+\mathbf{Q}}-\epsilon_{v \mathbf{k}}\right) A_{v c \mathbf{k}}^{(S, \mathbf{Q})}+\sum_{v^{\prime} c^{\prime} \mathbf{k}^{\prime}} K_{v c \mathbf{k}, v^{\prime} c^{\prime} \mathbf{k}^{\prime}}(\mathbf{Q}) A_{v^{\prime} c^{\prime} \mathbf{k}^{\prime}}^{(S, \mathbf{Q})} \\
& =\Omega^{(S, \mathbf{Q})} A_{v c \mathbf{k}}^{(S, \mathbf{Q})}
\end{aligned}
$$

Here, $\Omega^{(S, \mathbf{Q})}$ are the exciton energies and $A_{v c \mathbf{k}}^{(S, \mathbf{Q})}$ the amplitudes. The dependence on $Q$ enters via the singleparticle energies and the electron-hole interaction kernel $K_{v c \mathbf{k}, v^{\prime} c^{\prime} \mathbf{k}^{\prime}}(\mathbf{Q})$. This kernel includes the screened direct as well as the exchange interaction. The real-space exciton wave functions can be calculated by

$$
\Phi^{(S, \mathbf{Q})}\left(x_{h}, x_{e}\right)=\sum_{v, c, \mathbf{k}} A_{v, c, \mathbf{k}}^{(S, \mathbf{Q})} \phi_{v, \mathbf{k}}^{*}\left(x_{h}\right) \phi_{c, \mathbf{k}+\mathbf{Q}}\left(x_{e}\right)
$$

and their amplitude in the excitation spectra is given by the generalized dipole operator

$$
\begin{aligned}
\left\langle 0\left|e^{-i \mathbf{Q r}} \hat{\mathbf{p}}\right| S, \mathbf{Q}\right\rangle= & \sum_{v c \mathbf{k}} A_{v c \mathbf{k}}^{(S, \mathbf{Q})} \times \\
& \sum_{\sigma} \int \phi_{v, \mathbf{k}}^{*}(\mathbf{r}, \sigma) e^{-i \mathbf{Q r}} \hat{\mathbf{p}} \phi_{c, \mathbf{k}+\mathbf{Q}}(\mathbf{r}, \sigma) d \mathbf{r} .
\end{aligned}
$$

Note that the amplitude for nonzero momentum is not directly related to the optical absorbance.

The $G W+$ BSE method has previously been shown to yield excellent agreement with experiments [26 29]. While the absolute accuracy has been found to be about $0.1 \mathrm{eV}$ the relative accuracy of different excitons is typically $10 \mathrm{meV}$ or less [20, 22].

To investigate the entire exciton landscape all possible momenta $\mathbf{Q}$ in the irreducible part of first Brillouin zone need to be taken into account. However, as we are interested in the low energy excitations only, the investigation of momentum transfer between regions close to the band extrema is sufficient. In this study we consider $\mathbf{Q} \equiv(0,0, Q)$ with $Q=0 \ldots \overline{\Gamma K^{\prime}}$ as shown in Fig. 1 (a).

This choice is sufficient to cover all momenta relevant for the low-energy excitations. As $\mathbf{Q}$ is a vector connecting the valence and conduction band, the highlighted momentum of $\sim \frac{1}{2} \overline{\Gamma K^{\prime}}$ can lead to transitions for instance from $\Gamma \rightarrow \Lambda^{\prime}, \mathrm{K}^{\prime} \rightarrow \mathrm{M}$, or $\mathrm{K} \rightarrow \Lambda$. The latter one is shown inverted to $-Q$ in Fig. 1(b) which depicts the band structure of a monolayer of $\mathrm{MoS}_{2}$ along $\Gamma \rightarrow \mathrm{K}^{\prime} \rightarrow \mathrm{K}$.

\section{TMDC MONOLAYERS}

After having recapitulated the employed methodology we discuss the results for the four most common TMDC monolayers. In Fig. 2 the calculated exciton spectra for the $\mathrm{MoS}_{2}$ monolayer are shown for several different momenta $Q$. At the bottom the well known case of $Q \approx 0$ is depicted corresponding to the optical absorption spectrum. It contains the prominent spin-orbit split so called $\mathrm{A}$ and $\mathrm{B}$ excitons. All the energetically low lying excitations correspond to transitions at $\mathrm{K}$ and $\mathrm{K}^{\prime}$, i.e. $\mathrm{K} \rightarrow \mathrm{K}$ and $\mathrm{K}^{\prime} \rightarrow \mathrm{K}^{\prime}$. With increasing momentum $Q$ the excitons raise in energy and their oscillator strength rapidly decreases. We note that this strong decrease is caused by the varying orbital character of the valence and conduction bands which enter quadratically. Close to the middle of the plot the oscillator strength start to rise again and an excitation just above the $\mathrm{A}$ exciton becomes vis-

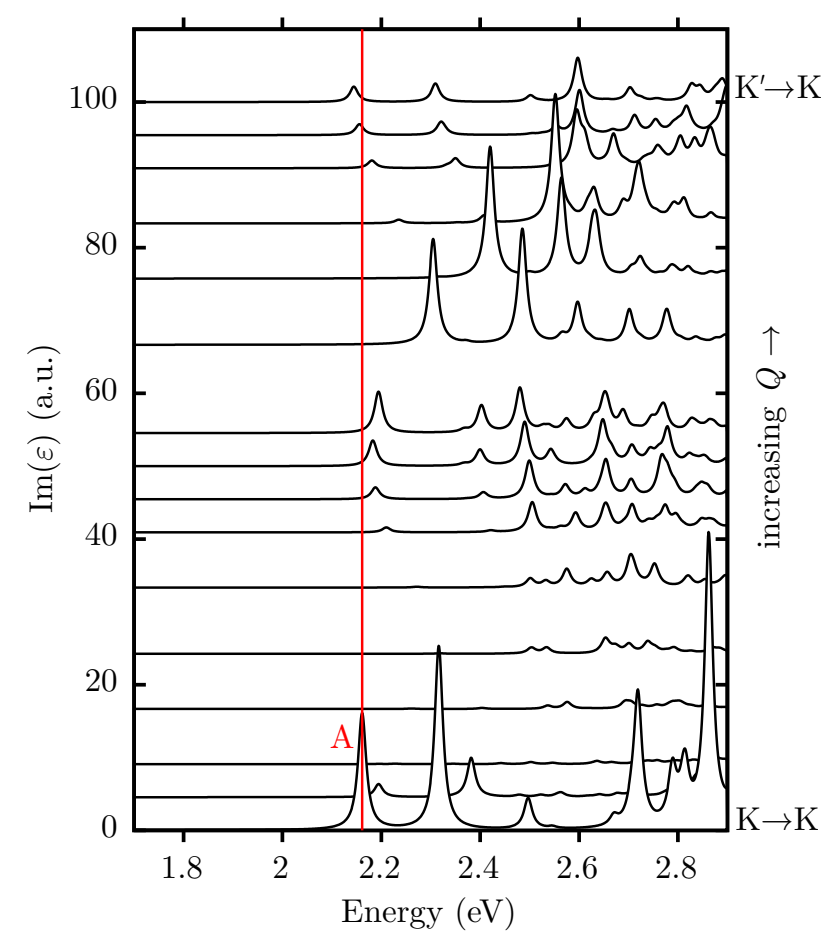

FIG. 2. Momentum dependent exciton spectra for the monolayer $\mathrm{MoS}_{2}$. The bottom spectrum shows the results for $Q \approx 0$ (i.e. $\mathrm{K} \rightarrow \mathrm{K}$ and $\mathrm{K}^{\prime} \rightarrow \mathrm{K}^{\prime}$ ). With increasing momentum $Q$ the offset is increased. The red line marks the energy of the optical bright A exciton. 
ible. In this exciton we find the hole in the valence band maximum at $\mathrm{K}$ and the electron at the minimum in the conduction band at the $\Lambda$ point. Close to the top of the plot an excitation with small oscillator strength shows up with a similar energy as the A exciton. This is an exciton with a hole in the valence band maximum at $\mathrm{K}^{\prime}$ and the electron in the conduction band minimum at $\mathrm{K}$.

Due to the different spin polarization of the split valence and conduction bands, the transitions at $\pm \mathrm{K}(Q=$ $0)$ can be selectively excited with circular polarized light [9. In the direction $\mathrm{K} \rightarrow \Gamma$ the spin polarization of the bands remains equally strong, e.g. at $\Lambda$. However, the excitation/decay of the $Q \neq 0$ excitons relies on an additional momentum transfer (e.g. by phonons). The details of this transfer process can strongly influence the valley selectivity of the calculated excitations.

To be able to track all excitations with vanishing and nonzero expectation value of the dipole operator, we turn to the representation of an "exciton band structure" in Fig. 3(a).

For each momentum, the exciton peaks are represented by a dot with the color of the dots denoting the strength of the dipole operator, i.e. red correspond to excitons with high oscillator strengths while black corresponds to excitons with vanishing oscillator strength. For $\mathrm{MoS}_{2}$ we can clearly identify minima at $Q=0$ and $Q=2 / 3$ (we use reciprocal units, i.e. $2 \pi / a_{\text {lat }}$, if not explicitly stated differently). We find that the transition $\mathrm{K} \rightarrow \mathrm{K}^{\prime}$ is about $30 \mathrm{meV}$ lower than the optically bright $\mathrm{A}$ exciton, and thus also $10 \mathrm{meV}$ below the lowest dark exciton at $Q=0$. We note that the ordering agrees well with the previous results of Qiu et al. which reported a difference of $5 \mathrm{meV}$ for the lowest state with momentum $Q=2 / 3$ [11.

In Fig. 3(b-d) similar plots are shown for the other TMDC monolayers $\mathrm{WS}_{2}, \mathrm{MoSe}_{2}$, and $\mathrm{WSe}_{2}$. The general trend is the same for all materials, namely we find a parabolic dispersion with minima at $Q=0,1 / 3$, and $2 / 3$. These characteristic shapes of the exciton band structure are largely inherited from the underlying single-particle band structures with minima at $\mathrm{K}, \Lambda$, and $\Gamma$. However, a more detailed view highlights some important differences. While $\mathrm{MoSe}_{2}$ shows a similar ordering compared to $\mathrm{MoS}_{2}$ the $Q=2 / 3$ excitation is $40 \mathrm{meV}$ below that at $Q=0$ while $Q=1 / 3$ is $40 \mathrm{meV}$ above. On the other hand, for $\mathrm{WX}_{2}$ all three minima are energetically much closer. This is a consequence of the smaller difference of the single-particle energies at $\mathrm{K}$ and $\Lambda$, i.e. the exciton binding energies var only little with $Q$. A detailed comparison of the energy differences is shown in Tab. I 30. We emphasize that the lowest energy transitions refer to zero momentum excitations with electrons and holes located at $\mathrm{K} / \mathrm{K}^{\prime}$. For $Q=1 / 3$ we find a further transition from $\Gamma \rightarrow \Lambda^{\prime}$ in addition to the lowest transition from $\mathrm{K} \rightarrow \Lambda$. For the monolayer this transition is higher in energy, however, this will change for the case of the bilayers discussed in Sec. IV. We note that the band gaps and exciton binding energies obtained here with the $G d W$ approximation quantitatively are larger compared to those
TABLE I. Direct gap at K, energy difference between $\mathrm{K}$ and $\Lambda$ (conduction band) $\Delta_{\mathrm{K}-\Lambda}^{\mathrm{CB}}$ and $\mathrm{K}$ and $\Gamma$ (valence band) $\Delta_{\mathrm{K}-\Gamma}^{\mathrm{VB}}$ for the four TMDC monolayers. The effective masses for holes $m_{h}$ and electron $m_{e}$ are given at $\mathrm{K}, \Lambda$, and $\Gamma$. Furthermore, the energy of optical bright A exciton, the dark-bright splitting $\Delta_{D A}$, and the exciton energies for $\mathrm{K} \rightarrow \Lambda$ and $\mathrm{K}^{\prime} \rightarrow \mathrm{K}$ are listed.

\begin{tabular}{ccccc}
\hline \hline TMDC & $\mathrm{MoS}_{2}$ & $\mathrm{WS}_{2}$ & $\mathrm{MoSe}_{2}$ & $\mathrm{WSe}_{2}$ \\
\hline $\mathrm{Gap}$ at $\mathrm{K}(\mathrm{eV})$ & 2.90 & 2.81 & 2.60 & 2.40 \\
$\Delta_{\mathrm{KB}}^{\mathrm{KB}}(\mathrm{meV})$ & 40 & 10 & 20 & -10 \\
$\Delta_{\mathrm{K}-\Gamma}^{\mathrm{B}}(\mathrm{meV})$ & 410 & 610 & 610 & 810 \\
$m_{h}$ at $\mathrm{K}\left(m_{0}\right)$ & 0.53 & 0.34 & 0.55 & 0.34 \\
$m_{h}$ at $\Gamma\left(m_{0}\right)$ & 4.28 & 3.09 & 9.54 & 4.16 \\
$m_{e}$ at $\mathrm{K}\left(m_{0}\right)$ & 0.48 & 0.36 & 0.52 & 0.38 \\
$m_{e}$ at $\Lambda\left(m_{0}\right)$ & 0.58 & 0.54 & 0.60 & 0.42 \\
\hline $\mathrm{A}$ " $\mathrm{K} \rightarrow \mathrm{K}(\mathrm{eV})$ & 2.16 & 2.17 & 1.80 & 1.78 \\
$\Delta_{D A}(\mathrm{meV})$ & 20 & 60 & 10 & 70 \\
$\mathrm{~K} \rightarrow \Lambda(\mathrm{eV})$ & 2.18 & 2.11 & 1.83 & 1.72 \\
$\mathrm{~K}^{\prime} \rightarrow \mathrm{K}(\mathrm{eV})$ & 2.13 & 2.12 & 1.75 & 1.72 \\
\hline \hline
\end{tabular}

in our recent $G W$ study [29]. However, the qualitative features such as the relative variation in energies with $Q$ are in good agreement. For a detailed comparison and further discussions of the accuracy of the $G d W$ method we refer to Ref. [20].

We stress that for $Q \rightarrow 0$ we find an increased slope of the exciton dispersion, but we do not observe a linear dispersion for the bright exciton as found in Refs. 11, 12. However, this deviation can be expected due to the fact that we do not truncate the Coulomb interaction (in 3D $\left.\propto 1 / Q^{2}\right)$, which is required to simulate a truly isolated $2 \mathrm{D}$ layer $(\propto 1 / Q)$. On the other hand, in practice $2 \mathrm{D}$ materials will be placed on a substrate, i.e. the Coulomb interaction is not strictly $2 \mathrm{D}$ anyway.

In the next step we analyse the influence of the electron-hole interaction on the exciton band structure. We pick the example of $\mathrm{WS}_{2}$ (from Fig. 3(b)) and compare to the bare transition energies between valence and conduction bands (green dashed lines, corresponds to zero electron-hole interaction) in Fig. 44. For better comparison the latter is shifted by $0.7 \mathrm{eV}$ (i.e. by the exciton binding energy) to match the energetically lowest dark exciton at $Q=0$. The bare transition energies show similar trends as the previously discussed exciton dispersions. The lowest transitions have a quadratic shape close to all three extrema. For all minima the influence of the electron-hole interaction is almost the same, i.e. the excitons experience a similar exciton binding energy independent of $Q$.

In previous $G W+\mathrm{BSE}$ calculations of TMDCs, the band gap and excitonic energies have been found to depend quite sensitively on the lattice constant 31, 32. Here we compare the influence of different lattice constants on the momentum dependent spectra, see Fig. 5 . While the previous results for $\mathrm{WS}_{2}$ (Fig. 3(b)) have been calculated for the experimental lattice constant, we show the results for our theoretical optimized lattice constant 
(a)
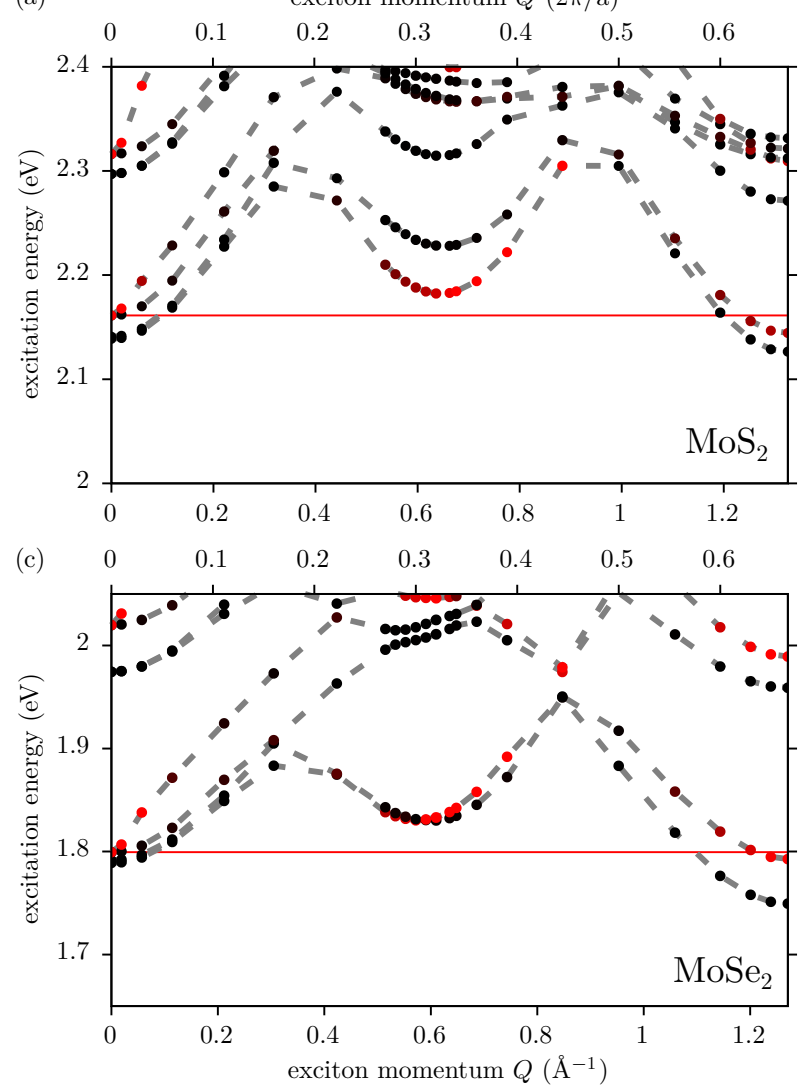

(b)

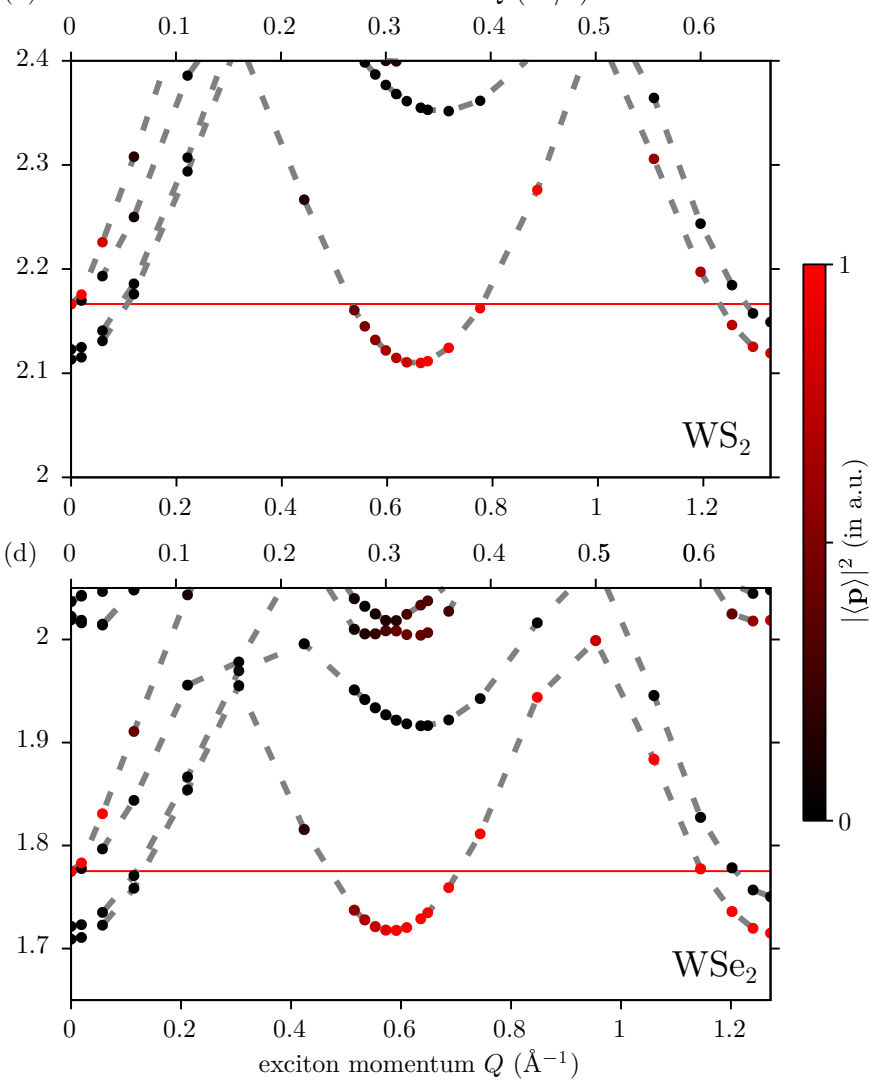

FIG. 3. Exciton band structure of monolayer $\mathrm{MoS}_{2}, \mathrm{WS}_{2}, \mathrm{MoSe}_{2}$, and $\mathrm{WSe}_{2}$. The grey dashed lines are a guide to the eye for the dots which denote the calculated peaks. The color corresponds to the expectation value of the dipole operator ranging from red (high amplitude) to black (zero amplitude). The horizontal red line shows the energy of the lowest bright A exciton $(Q=0)$.

(in LDA) in blue. The results are shifted down by $30 \mathrm{meV}$ for an easier comparison. While most transitions at $Q=0$ are only rigidly shifted those at $Q=1 / 3$ have moved down relatively by $30 \mathrm{meV}$. This is a result of the modified band structure at $\Lambda$ which is very sensitive to the lattice constant.

To get a more general picture of the dependency of the excitons on the applied strain in $\mathrm{WS}_{2}$, we evaluate strain dependent QP band structures and exciton spectra. The resulting trends for biaxial strain between -2 and $2 \%$ are shown in Fig. 6. For this small compression/expansion we find a nearly perfect linear response of the band gap at $\mathrm{K}$ and between $\mathrm{K}-\Lambda$. The corresponding deformation potentials are in good agreement with previous results [29. 33. Interestingly, the trends are reversed in sign and the changes at $\mathrm{K}$ are larger by about a factor of 3 . For the three excitons shown $(Q=0,1 / 3$, and $2 / 3)$ we find that they closely follow the behaviour of the band gap at the point with the largest contributions to the exciton. That means that the exciton binding energy is nearly constant. This results in an exciton ground state at $\mathrm{K}$ for the experimental lattice constant as well as for expanded $\mathrm{WS}_{2}$. On the other hand, the indirect $Q=1 / 3$ exciton is lower in energy if the material is compressed.
This behaviour could be interesting for pressure/strain sensors and similar applications 34.

In Ref. 15 Malic et al. have applied a Wannier model to describe the excitations close to the minima at $Q=0$, $1 / 3(\Lambda)$, and $2 / 3(\mathrm{~K})$. For $\operatorname{Mo} X_{2}$ we find $Q=2 / 3$ excitons to be lowest in energy. In contrast to this, the excitons at $Q=0$ and $2 / 3$ are at similar energies in [15] due to the neglected exchange in the Wannier model. For $\mathrm{W}_{2}$ Malic et al. observe distinctly lower excitons at $Q=1 / 3$ (compared to $Q=0$ ). Within our ab-inito approach we cannot reproduce this finding, however, the exact relative alignment also depend on the chosen lattice constant (see Fig. 6.

In addition to a possible strain field, a substrate leads to an enhanced dielectric screening and a hybridization of monolayer and substrate states. While the dielectric screening strongly effects band gaps and the exciton binding energies in opposite directions, the excitons typically only undergo a small redshift 35. This shift is similar for all momenta $Q$ for excitons located around $\mathrm{K}$ and $\Lambda$ [36]. On the other hand, the hybridization can differ greatly for different $Q$. For instance the modification of the band structure due to hybridization with substrates or other $2 \mathrm{D}$ materials, is much larger at $\Gamma$ than at $\mathrm{K}$. In 


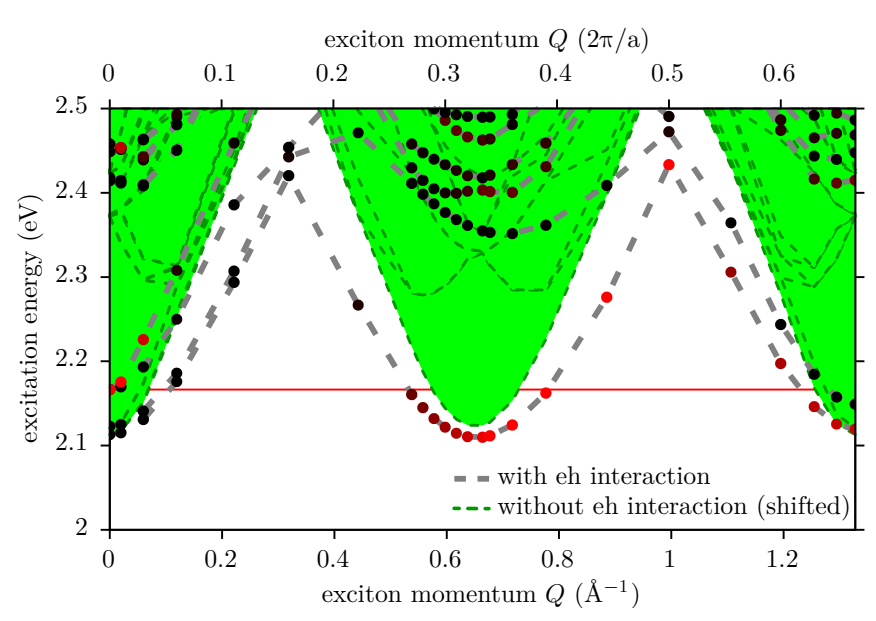

FIG. 4. Momentum dependent exciton spectrum of monolayer $\mathrm{WS}_{2}$. In addition to the previous result with electronhole interaction (grey dashed line and points) the resulting transitions without electron-hole interaction are shown as green dashed line. The latter have been shifted by the exciton binding energy of the lowest (dark) $Q=0$ exciton. In addition to the green bands which indicate possible transitions at the employed $k$-points, the green area indicates the continuous region of possible transitions for infinite $k$-points.

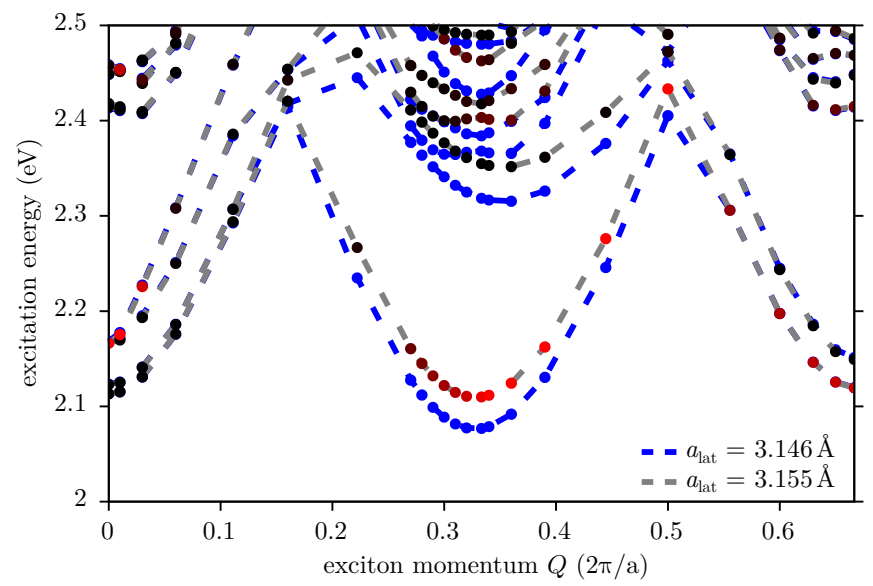

FIG. 5. Momentum dependent exciton spectrum of monolayer $\mathrm{WS}_{2}$ with different lattice constants. For an easier comparison the results using the theoretical lattice constant $\left(a_{\text {Lat }}=3.146 \AA\right.$, blue $)$ are shifted downward by $30 \mathrm{meV}$ to match the $Q=0$ result.

the next Sec. IV] we observe these effects for the bilayer materials. Due to the typically less perfect lattice match of the monolayer and a general substrate at the atomic level, the observed shifts in a bilayer should be seen as an upper limit to the hybridization in a supported or encapsulated monolayer.

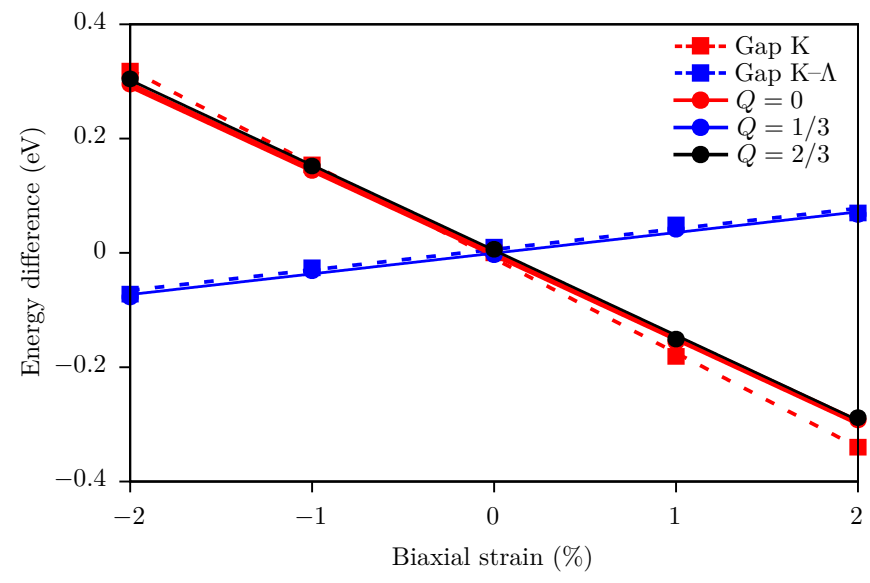

FIG. 6. Strain dependent changes of the QP gaps (relative to the minimal direct gap) and exciton energies (relative to the A exciton) in monolayer $\mathrm{WS}_{2}$. The QP gaps at $\mathrm{K}$ (red dashed line) and between $\mathrm{K}-\Lambda$ (blue dashed line) show a reversed trend. The resulting excitons at $Q=0,1 / 3$, and $2 / 3$ reciprocal units (red, blue, and black dots, respectively) closely follow the corresponding QP gaps. The shown lines are linear fits. Note that $Q=0$ is overlaid by $Q=2 / 3$.

\section{TMDC BILAYERS}

Now we turn to the discussion of the bilayers of $\mathrm{MoS}_{2}$, $\mathrm{WS}_{2}, \mathrm{MoSe}_{2}$, and $\mathrm{WSe}_{2}$. All four bilayer materials show clear indirect gaps (see Tab. II). Therefore, the bilayer materials are also prototypes for thicker multilayer systems up to the bulk.

Fig. 7 shows the momentum resolved exciton spectra for all four bilayers analogous to the monolayer spectra in Fig 3. A comparison of the excitations at $Q=0$ for bilayers and monolayers shows very similar results for the lowest energy excitations, which experience a small redshift of up to $60 \mathrm{meV}$ (for $\mathrm{WSe}_{2}$ ). The most obvious difference is observed around $Q \approx 1 / 3$ where the energies of the excitons are distinctly shifted downwards by up to $330 \mathrm{meV}$ (for $\mathrm{MoS}_{2}$ ). This shift is a consequence of the modified band structure which becomes indirect

TABLE II. Direct gap at $K$, energy difference $\Delta_{\mathrm{K}-\Lambda}^{\mathrm{CB}}$ and $\Delta_{\mathrm{K}-\Gamma}^{\mathrm{VB}}$ for the four TMDC bilayers. In addition to the energy of optical bright A exciton and the dark-bright splitting $\Delta_{D A}$ the energies for various possible transitions are listed.

\begin{tabular}{ccccc}
\hline \hline TMDC & $\mathrm{MoS}_{2}$ & $\mathrm{WS}_{2}$ & $\mathrm{MoSe}_{2}$ & $\mathrm{WSe}_{2}$ \\
\hline $\mathrm{Gap}$ at K $(\mathrm{eV})$ & 2.63 & 2.51 & 2.30 & 2.18 \\
$\Delta_{\mathrm{K}-\Lambda}^{\mathrm{CB}}(\mathrm{meV})$ & -190 & -140 & -150 & -170 \\
$\Delta_{\mathrm{K}-\Gamma}^{\mathrm{VB}}(\mathrm{meV})$ & -130 & 90 & 10 & 280 \\
\hline "A" K $\rightarrow \mathrm{K}(\mathrm{eV})$ & 2.12 & 2.10 & 1.76 & 1.76 \\
$\Delta_{D A}(\mathrm{meV})$ & 20 & 50 & 10 & 60 \\
$\mathrm{~K} \rightarrow \Lambda(\mathrm{eV})$ & 2.00 & 1.94 & 1.70 & 1.58 \\
$\Gamma \rightarrow \Lambda(\mathrm{eV})$ & 1.84 & 1.99 & 1.70 & 1.82 \\
$\mathrm{~K}^{\prime} \rightarrow \mathrm{K}(\mathrm{eV})$ & 2.09 & 2.06 & 1.72 & 1.71 \\
$\Gamma \rightarrow \mathrm{K}(\mathrm{eV})$ & 2.00 & 2.15 & 1.82 & 1.98 \\
\hline \hline
\end{tabular}


(a)

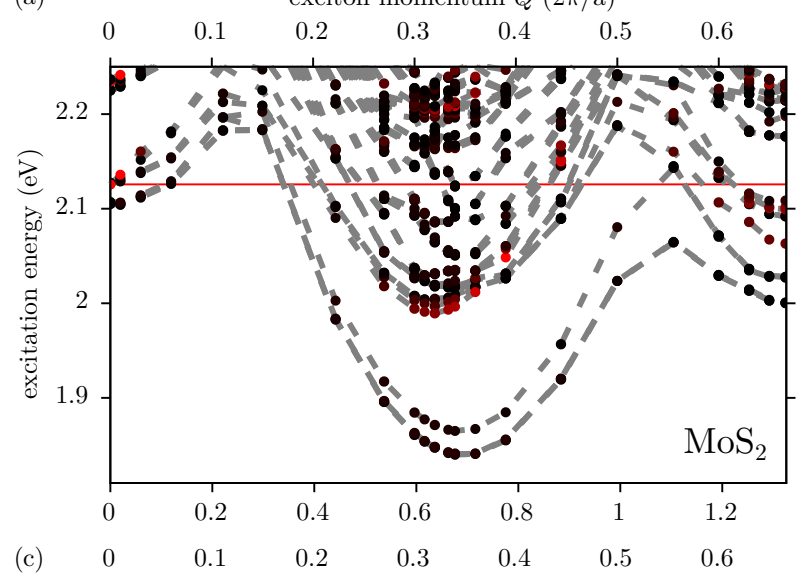

(c)

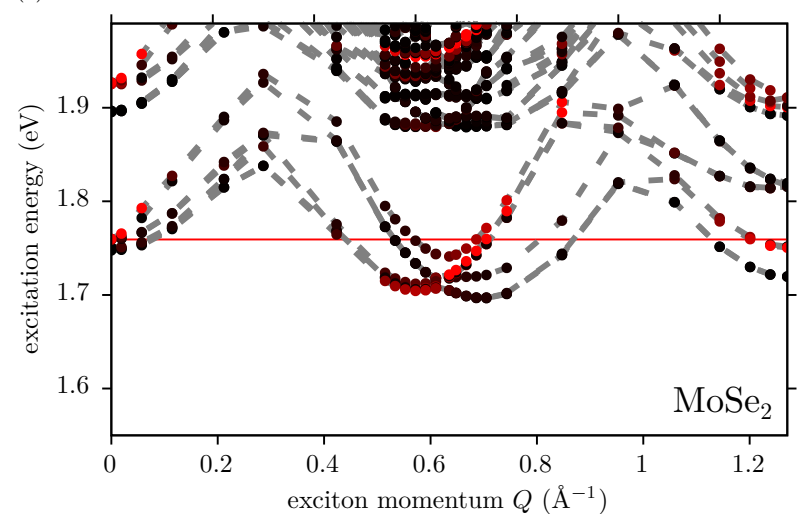

(b)

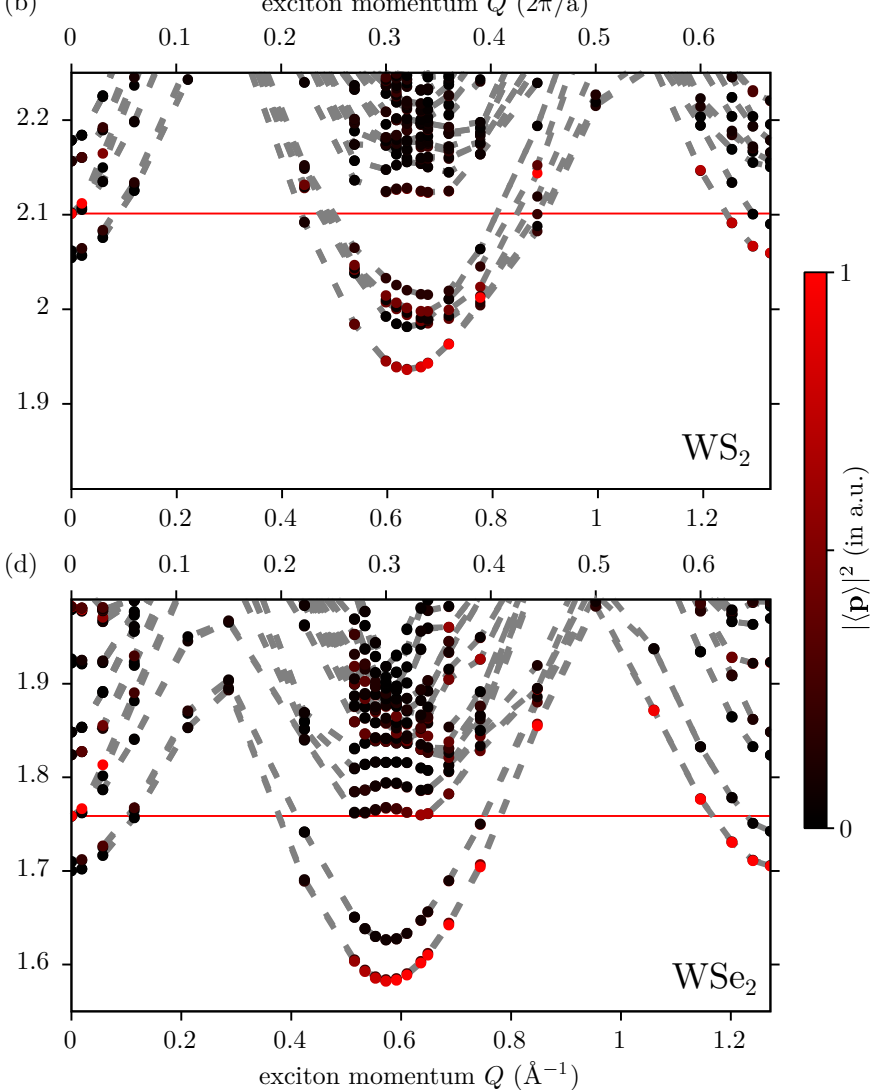

FIG. 7. Exciton band structure of bilayer $\mathrm{MoS}_{2}, \mathrm{WS}_{2}, \mathrm{MoSe}_{2}$, and $\mathrm{WSe}_{2}$. The horizontal red line shows the energy of the lowest bright A exciton, see caption of Fig. 3

(150-300 meV, see Tab. II). E.g. for $\mathrm{MoS}_{2}$ we find an A exciton energy of $2.12 \mathrm{eV}$ while the energetically lowest exciton is found at $1.84 \mathrm{eV}$ for $\Gamma \rightarrow \Lambda$. The energy difference of the excitions is nearly identical to the difference between the direct and indirect band gaps. Beyond this general tendency for $Q \approx 1 / 3$ the details are different for all materials. In $\mathrm{MoS}_{2}$ and $\mathrm{MoSe}_{2}$ the $\Gamma \rightarrow \Lambda$ excitation is lowest in energy. This is most pronounced for $\mathrm{MoS}_{2}$ for which the valence band at $\Gamma$ is $130 \mathrm{meV}$ higher in energy compared to $\mathrm{K}$. On the other hand, for $\mathrm{WS}_{2}$ and $\mathrm{WSe}_{2}$ the $\mathrm{K} \rightarrow \Lambda$ transitions are energetically lowest. We note that we find a vanishing oscillator strength for $\Gamma \rightarrow \Lambda$ excitons while it is nonzero for $\mathrm{K} \rightarrow \Lambda$. The latter oscillator strengths are similar to those found in monolayers.

A further special feature is observed at $Q=2 / 3$ for $\mathrm{MoS}_{2}$. While for all other materials the energetically lowest excitation occurs from $\mathrm{K}^{\prime} \rightarrow \mathrm{K}$ the strong hybridization of the valence band at $\Gamma$ leads to a lower $\Gamma \rightarrow \mathrm{K}$ transition by about $90 \mathrm{meV}$.

Analogous to the investigation of strain induced effects for monolayers (Fig. 6) we investigate the bilayer $\mathrm{WS}_{2}$ in Fig. 8. In agreement with previous studies based on DFT (see e.g. [37, 38] and the references therein), we find that bilayer $\mathrm{WS}_{2}$ has an indirect gap. Close to $0.7 \%$ biaxial strain the $\Gamma-\mathrm{K}$ gap drops below the $\mathrm{K}-\Lambda$ gap. As observed for the monolayer, the exciton energies closely

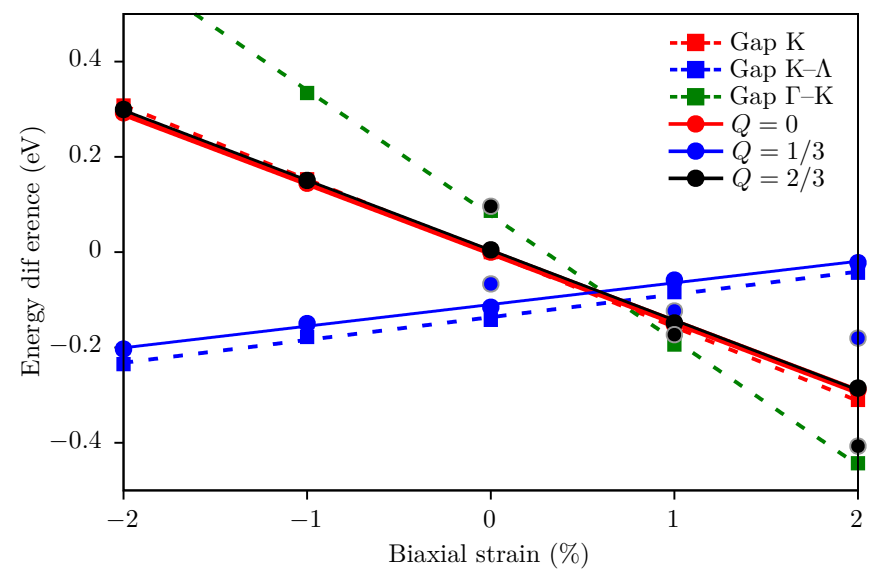

FIG. 8. Strain dependent changes of the QP gaps (relative to the minimal direct gap) and exciton energies (relative to the A exciton) in bilayer $\mathrm{WS}_{2}$. The colors are chosen similar to Fig. 8. Note that the additional excitations (blue and black dots which do not follow the lines) represent excitations from $\Gamma \rightarrow \Lambda$ and $\Gamma \rightarrow \mathrm{K}$, respectively.

follow the trends of the related gaps, i.e. the exciton binding energies are almost independent of the applied strain. 


\section{CONCLUSIONS}

We have studied the exciton band structure of the four prototypical transition metal dichalcogenides employing many-body perturbation theory within the $G d W / \mathrm{BSE}$ approximation. Throughout the entire $Q$-space strong electron-hole interactions are present which do not vary significantly. This results in an exciton ground state with $\mathrm{K} \rightarrow \mathrm{K}$ ' transitions for $\mathrm{MoX}_{2}$ monolayers and zero momentum transitions for $\mathrm{WX}_{2}$ monolayers. For bilayers the lowest energy transitions are shifted to $Q=\Lambda$ closely following the trends of the single-particle band struc- tures. At the same time, the coupling strength varies significantly depending on the character of the corresponding wave functions and $Q$.

\section{Acknowledgements}

T.D. acknowledges financial support from the Villum foundation and fruitful discussions with Ashish Arora and Michael Rohlfing. The Center for Nanostructured Graphene is sponsored by the Danish National Research Foundation, Project DNRF103. This project has received funding from the European Research Council (ERC) under the European Union's Horizon 2020 research and innovation programme (grant agreement No 773122, LIMA).
[1] Andrea C Ferrari, Francesco Bonaccorso, Vladimir FalḰo, Konstantin S Novoselov, Stephan Roche, Peter Bøggild, Stefano Borini, Frank HL Koppens, Vincenzo Palermo, Nicola Pugno, et al., "Science and technology roadmap for graphene, related two-dimensional crystals, and hybrid systems," Nanoscale 7, 4598-4810 (2015).

[2] Ganesh R Bhimanapati, Zhong Lin, Vincent Meunier, Yeonwoong Jung, Judy Cha, Saptarshi Das, Di Xiao, Youngwoo Son, Michael S Strano, Valentino R Cooper, et al., "Recent advances in two-dimensional materials beyond graphene," Acs Nano 9, 11509-11539 (2015)

[3] Kin Fai Mak, Changgu Lee, James Hone, Jie Shan, and Tony F. Heinz, "Atomically Thin MoS 2 : A New Direct-Gap Semiconductor," Physical Review Letters 105, 136805 (2010)

[4] Andrea Splendiani, Liang Sun, Yuanbo Zhang, Tianshu Li, Jonghwan Kim, Chi-Yung Chim, Giulia Galli, and Feng Wang, "Emerging Photoluminescence in Monolayer MoS 2," Nano Letters 10, 1271-1275 (2010)

[5] Kin Fai Mak, Keliang He, Jie Shan, and Tony F. Heinz, "Control of valley polarization in monolayer MoS2 by optical helicity," Nature Nanotechnology 7, 494-498 (2012)

[6] G. Sallen, L. Bouet, X. Marie, G. Wang, C. R. Zhu, W. P. Han, Y. Lu, P. H. Tan, T. Amand, B. L. Liu, and B. Urbaszek, "Robust optical emission polarization in $\mathrm{MoS}_{2}$ monolayers through selective valley excitation," Phys. Rev. B 86, 081301 (2012)

[7] Hualing Zeng, Junfeng Dai, Wang Yao, Di Xiao, and Xiaodong Cui, "Valley polarization in MoS2 monolayers by optical pumping," Nature Nanotechnology 7, 490-493 (2012).

[8] T. Cao, G. Wang, W. Han, H. Ye, C. Zhu, J. Shi, Q. Niu, P. Tan, E. Wang, B. Liu, and J. Feng, "Valleyselective circular dichroism of monolayer molybdenum disulphide," Nature Communications 3, 887 (2012)

[9] Di Xiao, Gui-Bin Liu, Wanxiang Feng, Xiaodong Xu, and Wang Yao, "Coupled Spin and Valley Physics in Monolayers of MoS 2 and Other Group-VI Dichalcogenides," Physical Review Letters 108, 196802 (2012).

[10] Hannah Catherine Nerl, Kirsten Trøstrup Winther, Fredrik S. Hage, Kristian Sommer Thygesen, Lothar Houben, Claudia Backes, Jonathan N. Coleman, Quentin M. Ramasse, and Valeria Nicolosi, "Probing the local nature of excitons and plasmons in few-layer mos2," npj 2D Materials and Applications 1, 2 (2017).
[11] Diana Y. Qiu, Ting Cao, and Steven G. Louie, "Nonanalyticity, Valley Quantum Phases, and Lightlike Exciton Dispersion in Monolayer Transition Metal Dichalcogenides: Theory and First-Principles Calculations," Physical Review Letters 115, 176801 (2015)

[12] Pierluigi Cudazzo, Lorenzo Sponza, Christine Giorgetti, Lucia Reining, Francesco Sottile, and Matteo Gatti, "Exciton Band Structure in Two-Dimensional Materials," Physical Review Letters 116, 066803 (2016)

[13] A. Steinhoff, M. Florian, M. Rösner, G. Schönhoff, T. O. Wehling, and F. Jahnke, "Exciton fission in monolayer transition metal dichalcogenide semiconductors," Nature Communications 8, 1166 (2017)

[14] Maja Feierabend, Gunnar Berghäuser, Andreas Knorr, and Ermin Malic, "Proposal for dark exciton based chemical sensors," Nature Communications 8, 14776 (2017).

[15] Ermin Malic, Malte Selig, Maja Feierabend, Samuel Brem, Dominik Christiansen, Florian Wendler, Andreas Knorr, and Gunnar Berghäuser, "Dark excitons in transition metal dichalcogenides," Physical Review Materials 2, 014002 (2018).

[16] Iris Niehues, Robert Schmidt, Matthias Drüppel, Philipp Marauhn, Dominik Christiansen, Malte Selig, Gunnar Berghäuser, Daniel Wigger, Robert Schneider, Lisa Braasch, Rouven Koch, Andres Castellanos-Gomez, Tilmann Kuhn, Andreas Knorr, Ermin Malic, Michael Rohlfing, Steffen Michaelis de Vasconcellos, and Rudolf Bratschitsch, "Strain control of exciton-phonon coupling in atomically thin semiconductors," Nano Letters 18, 1751-1757 (2018)

[17] D. R. Hamann, "Generalized norm-conserving pseudopotentials," Physical Review B 40, 2980-2987 (1989).

[18] Leonard Kleinman and D. M. Bylander, "Efficacious Form for Model Pseudopotentials," Physical Review Letters 48, 1425-1428 (1982).

[19] Thorsten Deilmann, Peter Krüger, Michael Rohlfing, and Daniel Wegner, "Adsorption and STM imaging of tetracyanoethylene on $\mathrm{Ag}(001)$ : An ab initio study," Physical Review B 89, 045405 (2014)

[20] Matthias Drüppel, Thorsten Deilmann, Jonathan Noky, Philipp Marauhn, Peter Krüger, and Michael Rohlfing, "Electronic excitations in transition metal dichalcogenide monolayers from an LDA + GdW approach," Phys. Rev. B 98, 155433 (2018)

[21] Lars Hedin, "New Method for Calculating the One- 
Particle Green's Function with Application to the Electron-Gas Problem," Phys. Rev. 139, A796-A823 (1965)

[22] Michael Rohlfing, "Electronic excitations from a perturbative LDA+GdW approach," Physical Review B 82, 205127 (2010)

[23] G. Strinati, "Dynamical Shift and Broadening of Core Excitons in Semiconductors," Physical Review Letters 49, 1519-1522 (1982).

[24] Michael Rohlfing and Steven G. Louie, "Electron-hole excitations and optical spectra from first principles," Physical Review B 62, 4927-4944 (2000)

[25] Giovanni Onida, Lucia Reining, and Angel Rubio, "Electronic excitations: Density-functional versus many-body Green's-function approaches," Rev. Mod. Phys. 74, 601659 (2002)

[26] Michael Rohlfing and Steven G. Louie, "Electron-Hole Excitations in Semiconductors and Insulators," Physical Review Letters 81, 2312-2315 (1998)

[27] Michael Rohlfing and Steven G. Louie, "Optical Excitations in Conjugated Polymers," Physical Review Letters 82, 1959-1962 (1999).

[28] Catalin D. Spataru, Sohrab Ismail-Beigi, Lorin X. Benedict, and Steven G. Louie, "Excitonic Effects and Optical Spectra of Single-Walled Carbon Nanotubes," Physical Review Letters 92, 077402 (2004).

[29] Sten Haastrup, Mikkel Strange, Mohnish Pandey, Thorsten Deilmann, Per S. Schmidt, Nicki F. Hinsche, Morten N. Gjerding, Daniele Torelli, Peter M. Larsen, Anders C. Riis-Jensen, Jakob Gath, Karsten W. Jacobsen, Jens Jørgen Mortensen, Thomas Olsen, and Kristian S. Thygesen, "The computational 2d materials database: High-throughput modeling and discovery of atomically thin crystals," 2D Materials 5, 042002 (2018).

[30] We note that the error bars for the determination of the effective masses are at least $\pm 10 \%$.

[31] Won Seok Yun, S. W. Han, Soon Cheol Hong, In Gee Kim, and J. D. Lee, "Thickness and strain effects on electronic structures of transition metal dichalcogenides: $2 \mathrm{H}-M X_{2}$ semiconductors $(M=\mathrm{Mo}, \mathrm{W} ; X=\mathrm{S}, \mathrm{Se}, \mathrm{Te})$," Physical Review B 85, 033305 (2012).

[32] Falco Hüser, Thomas Olsen, and Kristian S. Thygesen, "How dielectric screening in two-dimensional crystals affects the convergence of excited-state calculations: Monolayer MoS 2," Physical Review B 88, 245309 (2013).

[33] Julia Wiktor and Alfredo Pasquarello, "Absolute deformation potentials of two-dimensional materials," Phys. Rev. B 94, 245411 (2016).

[34] Lei Fu, Yi Wan, Ning Tang, Yi-min Ding, Jing Gao, Jiachen Yu, Hongming Guan, Kun Zhang, Weiying Wang, Caifeng Zhang, and et al., "K- crossover transition in the conduction band of monolayer mos 2 under hydrostatic pressure," Science Advances 3, e1700162 (2017)

[35] Matthias Drüppel, Thorsten Deilmann, Peter Krüger, and Michael Rohlfing, "Diversity of trion states and substrate effects in the optical properties of an $\operatorname{mos}_{2}$ monolayer," Nature Communications 8, 2117 (2017)

[36] The valence band close to $\Gamma$ is influenced a little more and thus the redshift of such excitons is slightly stronger.

[37] Hiram J. Conley, Bin Wang, Jed I. Ziegler, Richard F. Haglund, Sokrates T. Pantelides, and Kirill I. Bolotin, "Bandgap Engineering of Strained Monolayer and Bilayer $\mathrm{MoS}_{2}, "$ Nano Letters 13, 3626-3630 (2013).

[38] Luciano Ortenzi, Luciano Pietronero, and Emmanuele Cappelluti, "Zero-point motion and direct-indirect bandgap crossover in layered transition-metal dichalcogenides," Physical Review B 98, 195313 (2018). 